\title{
Inhaltsverzeichnis
}

Christa Dürscheid/Jan Georg Schneider

Einleitung — IX

I Grundfragen

Christian Stetter

1. System und Performanz -3

Arnulf Deppermann/Nadine Proske

2. Grundeinheiten der Sprache und des Sprechens - 17

Ellen Fricke

3. Grammatik und Multimodalität -48

Clemens Knobloch/Josephine Krüger

4. Zum Erwerb syntaktischer Konstruktionen - 77

Renata Szczepaniak

5. Syntaktische Einheitenbildung - typologisch und diachron

betrachtet -104

Jan Georg Schneider

6. Syntaktische Schemabildung - zeichentheoretisch betrachtet -125

II Satz

Gisela Zifonun

7. Satz - oberflächennah 155

Margret Selting

8. Sentences and clauses - from the perspective of Interactional

Linguistics -180

Peter Öhl

9. Satz - aus Sicht der Generativen Grammatik — 205 
Mechthild Habermann

10. Satz in der Schulgrammatik -231

Klaus Welke

11. Satzaufbau und Verbvalenz -255

Mathilde Hennig

12. Ellipsen -279

\section{III Äußerung}

Frank Liedtke

13. Syntax und moderne Pragmatik -299

Sven Staffeldt

14. Einheiten des pragmatischen Standards - 326

Stephan Stein

15. Einheiten der gesprochenen und der geschriebenen Sprache - 345

Reinhard Fiehler

16. Syntaktische Phänomene in der gesprochenen Sprache - 370

Gisela Fehrmann

17. Einheitenbildung in der Deutschen Gebärdensprache - 396

Jana Bressem

18. Repetition als Mittel der Musterbildung bei redebegleitenden Gesten -422

\section{Schema}

Jörg Bücker

19. Schema - Muster - Konstruktion - 445

Susanne Tienken

20. Muster - kulturanalytisch betrachtet -464

Noah Bubenhofer

21. Muster aus korpuslinguistischer Sicht -485 
Alexander Lasch

22. Konstruktionen in der geschriebenen Sprache - 503

Georg Albert

23. Konstruktionen in unterschiedlichen medialen Kontexten -527

Wolfgang Imo

24. Was ist (k)eine Konstruktion? -551

Sachregister $\longrightarrow \mathbf{5 7 7}$ 
